\title{
Applications of Nature-Inspired Intelligence in Finance
}

\author{
Vasilios Vasiliadis, and Georgios Dounias \\ University of the Aegean, Dept. of Financial Engineering and \\ Management, Management \& Decision Engineering Laboratory, \\ 31 Fostini Str., GR-821 00, Chios, GREECE, \\ tel: +30-2271-0-35454(35483), fax: +30-2271-0-35499, \\ 23100045@fme.aegean.gr, g.dounias@aegean.gr, \\ WWW home page: http://decision.fme.aegean.gr
}

\begin{abstract}
A great variety of complex real-life problems can be sufficiently solved by intelligent nature-inspired methods which can be considered part of artificial or computational intelligence. These newly introduced techniques have proven their important role on many successful implementations, mostly related to optimization problems. The basic reason for their success is that they imitate the way that real-life networks and other biological systems function and evolve in order to solve problems in different domains. Such systems can be found in the human brain (neurons), or can be observed in the natural world in the form of ant colonies, flocks of birds, as well as in other examples taken from the microcosm such as the human immune system. In this paper, we try to briefly present popular nature-inspired techniques, ant colony optimization and particle swarm optimization, and also to clarify the significance and appropriateness of nature-inspired intelligent approaches for solving complex financial optimization problems. A short discussion in included for a number of selected financial decision making applications, i.e. forecasting of financial distress, multi-stage portfolio optimization, credit scoring, investment decisions and capital investment planning.
\end{abstract}

Please use the following format when citing this chapter:

Vasiliadis, V., Dounias, G., 2007, in IFIP International Federation for Information Processing, Volume 247, Artificial Intelligenee and Innovations 2007: From Theory to Applications, eds. Boukis, C., Pnevmatikakis, I.., Polymenakos, I.., (Boston: Springer), pp. 187-194. 


\section{Introduction}

In the recent years, both global economy and technology have advanced rapidly. A clear effect of this is the fact that new problem domains have been created, difficult to handle with standard approaches. New techniques are required, usually computer assisted, that offer effective and premium solutions with the least computational effort. However, it is indicative that new nature-inspired techniques have arose aiming at solving complex optimization problems. Nature-inspired intelligent systems mimic the way in which biological systems and real-world networks function in order to discover solutions in difficult optimization problems. Such examples are the function of the human brain (neural networks), the process through which an ant colony searches for its food, etc.

In literature there is a variety of engineering, medical, managerial or financial applications, suitable to be handled through nature-inspired approaches. In production and manufacturing, the key issue is usually to determine the optimal parameters in product planning. An important point is often the optimization of the time distributed in each workstation.

In medical domains, nature-inspired techniques are appropriate in making predictions for certain health problems, based on specific disease characteristics corresponding to decision variables. Finally, financial problems are of great importance. We deal with these kinds of problems later in this paper.

The present work mainly focuses on the description of two varieties of optimization algorithms. Their common characteristic is that both are based on the way in which biological systems behave.

The first method is ant colony optimization, an approach that mimics the way in which a colony of ants searches for its food. Ants search for food at random. After they have found a possible source, they return to their colony depositing pheromone in all the way. Other ants realize this chemical product. The power of pheromone is a function of the quality of the food and the distance between the nest and the source of food. The second algorithm is particle swarm optimization. It imitates the way in which a flock of birds migrates. Each bird has a certain speed and direction, as if a vector. All birds communicate with each other and finally decide to adopt the direction of the one that has the best position according to their final destination. In the next section, we depict in more detail the way that the two algorithmic approaches work in principle.

The paper is organized as follows: In section 2, a brief description of the optimization algorithms is given. In section 3, we present some of the financial applications successfully solved by these approaches. In the final section, we propose future trends and research perspectives of nature-inspired approaches, not only in the financial domain, but in other domains as well. 


\section{Popular nature-inspired optimization algorithms}

\subsection{Ant colony optimization}

Ant colony optimization (ACO) algorithms were first introduced by $\mathrm{M}$. Dorigo in the early 1990 's $[8,12,13]$. The development of these algorithms was inspired by the observation of ant colonies. Ants' behavior is governed by the goal of colony survival rather than being focused on the survival of individuals. The philosophy of these optimization algorithms stems from the ants' foraging behavior, and in particular, how ants can find shortest paths between food sources and their nest. When searching for food, ants initially explore the area surrounding their nest in a random manner. While moving, ants leave a chemical pheromone trail on the ground, which can be smelled by other members of the colony. Other ants tend to choose, in probability, paths marked by strong pheromone concentrations. As soon as an ant finds a food source, it evaluates the quantity and the quality of the food and carries some of it back to the nest. During the return trip, the quantity of pheromone that an ant leaves on the ground may depend on the quantity and quality of the food. These pheromone trails will guide other ants to the food source. It has been shown that the indirect communication between the ants via pheromone trails-known as stigmergy-enables them to find shortest paths between their nest and food sources.

In general, the ACO approach attempts to solve an optimization problem by iterating the following steps:

- Candidate solutions are constructed using a pheromone model, that is, a parameterized probability distribution over the solution space;

- The candidate solutions are used to modify the pheromone values in a way that is deemed to bias future sampling toward high quality solutions;

Finally, the pheromone update aims to concentrate the search in regions of the search space containing high quality solutions.

\subsection{Particle swarm optimization}

The first particle swarm optimization (PSO) algorithm was proposed by Kennedy and Eberhart in $1995[14,10]$. It is a biologically inspired algorithm which models the social dynamics of bird flocking. A large number of birds flock synchronously, change direction suddenly, scatter and regroup iteratively, and finally perch on a target. The PSO algorithm facilitates simple rules simulating bird flocking and serves as an optimizer for continuous nonlinear functions. The attractiveness of the PSO algorithm is due to features like natural metaphor, stochastic move, adaptivity, and positive feedback.

The general principles of the PSO algorithm are outlined as follows: 
- Particle representation: The particle in the PSO is a candidate solution to the underlying problem and move iteratively about to the solution space.

- Swarm: The PSO explores the solution space by flying a number of particles, called swarm.

- Personal best experience and swarm's best experience: The PSO enriches the swarm intelligence by storing the best positions visited so far by every particle. In particular, each particle remembers the best position among those it has visited and the best position by its neighbors.

- Particle movement: The PSO is an iterative algorithm according to which a swarm of particles flies about the solution space until the stopping criterion is satisfied.

Stopping criterion: The PSO algorithm is terminated with a maximal number of iterations or the best particle position of the entire swarm cannot be improved further after a sufficiently large number of iterations.

\section{Financial applications}

The aforementioned nature-inspired optimization algorithms have been recently (2006) used, among others, in a variety of applications of the financial domain. A first issue that has been studied is the forecasting of financial distress for companies [1]. Due to the radical changing of the global economy, a more precise forecasting of corporate financial distress helps provide important judgment principles to decisionmakers. Although financial statements reflect a firm's business activities, it is very challenging to discover critical information from these statements.

In this study, an evolutionary approach with modularized evaluation functions, which extracts the set of critical financial ratios and integrates more evaluation functions modules to achieve a better forecasting accuracy by assigning distinct weights, is introduced to forecast financial distress. PSO and genetic algorithms (GA) will be the evolutionary algorithms and logistic regression, discriminant analysis and neural networks will be the evaluation modules. In conclusion, it appears that the use of the proposed approach achieves better forecasting accuracy with a minimum critical consideration of financial ratios than using conventional statistical techniques. Furthermore, more integrated evaluation modules would achieve better forecasting accuracies in our approach.

Credit scoring is another issue of great importance both for the academic and the business society [2]. Numerous modeling techniques have been developed in order to tackle this problem. The use of the particle swarm optimization described above, introduces a quite original approach. The algorithm is used in order to train a neural network. This hybridized algorithm is used in order to evaluate the creditability of real-world cases. There are two advantages from this approach. Firstly, the neural network, which comes from the parameterization process of the optimization algorithm, has better decision making ability. Secondly, we can comment on the classification ability of the network, which is improved greatly. 
Another application is a multistage stochastic financial optimization problem [3]. Multistage stochastic financial optimization manages portfolio in constantly changing financial markets by periodically rebalancing the asset portfolio to achieve return maximization and/or risk minimization. Particle swarm optimization is used for the decision-making process in order to solve the multi-stage portfolio optimization problem. The performance of the algorithm is demonstrated by optimizing the allocation of cash and various stocks in a weighted index.

There are two more recent works based on the topic of portfolio optimization $[18,19]$. In [18], a particle swarm optimization algorithm was applied to the construction of optimal risky portfolios for financial investments. Constructing an optimal risky portfolio is a high-dimensional constrained optimization problem where financial investors look for an optimal combination of their investments among different financial assets with the aim of achieving a maximum reward-tovariability ratio (Sharpe ratio). A particle swarm solver is developed and tested on various restricted and unrestricted risky investment portfolios. The particle swarm solver demonstrates high computational efficiency in constructing optimal risky portfolios consisting of a small number of assets. In [19], apart from including stocks in the portfolio, bonds are also taken as potential assets for investment.

Another application of particle swarm optimization algorithm to financial problems is the construction of a decision making model that generates one-step forward investment decisions for stock markets. In this case, the optimization algorithm is hybridized with a neural network $[4,17,21]$. The neural network is used to make the analysis of daily stock returns and to calculate one day forward decision for the purchase of the stocks. Subsequently the PSO algorithm is applied in order to select the "best" ANN for the future investment decisions and to adapt the weights of other networks towards the weights of the best network. The experimental investigations were made considering different forms of decision-making model, i.e. different number of ANN, ANN inputs, sliding windows, and commission fees. One of the central problems in financial markets is to make the profitable stocks trading decisions using historical stocks' market data [21]. In order to achieve this, a decision making method, which is based on the application of neural networks and swarm intelligence technologies is presented. This model generates one-step-ahead investment decisions. In brief, the analysis of historical stock prices variations is made using single layer NN, and subsequently the Particle Swarm Optimization algorithm is applied in order to select global best $\mathrm{NN}$ for the future investment decisions and to adapt the weights of other networks towards the weights of the best network.

An application of the ant colony optimization algorithm for the capital investment planning problem is given in [7]. Capital investment planning is a periodic management task that is particularly challenging in the presence of multiple objectives as trade-offs have to be made with respect to the preferences of the decision-makers. The underlying mathematical model is a multi-objective combinatorial optimization problem. One way to tackle this problem is first to determine the set of all efficient portfolios and then to explore this set in order to identify a final preferred portfolio. The ant colony optimization algorithm is developed in order to find efficient portfolios as it is impossible to enumerate all of 
them within a reasonable computation time for practical problems. Firstly, a neighbourhood search routine is added to $\mathrm{ACO}$ to improve its performance. Then a taboo search and variable neighbourhood search procedure are developed. The multiobjective integer linear programming model of the capital investment planning problem is adopted. There are two sets of constraints in the model. The first set restricts each feasible portfolio to contain no more than a given maximum (or no less than a given minimum) number of projects out $f$ a certain subset. The second set of constraints deals with resource limitations. Results of this study indicated that the performance of ACO can be remarkably improved by supplementing it with a neighbourhood search routine. The improved ACO provides the best solution and is less parameter-sensitive than other procedures.

The topic of time series analysis and data processing in general is of great financial importance [6,15]. In [15], a recurrent neural network is trained with a new learning algorithm in order to predict a number of missing values from a certain time series. This training algorithm is based on a hybrid of particle swarm optimization and evolutionary algorithm. By combining the searching abilities of these two global optimization methods, the evolution of individuals is no longer restricted to be in the same generation, and better performing individuals may produce offspring to replace those with poor performance. The trained RNNs are able to predict the missing values in the time series with minimum error.

Another important issue in the field of financial management is the modeling of time series, as well as the prediction and forecasting ability of the model [16,22,23]. In [16], an attempt to represent the seemingly chaotic behavior of stock markets by using flexible neural networks is made. Again, the parameters of the neural network are optimized using genetic programming and particle swarm optimization algorithms. In [22], a time series forecasting methodology and its application to generate one-step-ahead predictions for two daily foreign exchange spot rate time series is presented. The methodology draws from the disciplines of chaotic time series analysis, clustering, artificial neural networks and evolutionary computation. In brief, clustering is applied to identify neighborhoods in the reconstructed state space of the system; and subsequently neural networks are trained to model the dynamics of each neighborhood separately. Again, the training techniques used are an evolutionary and a particle swarm optimization algorithm. Finally, in [23], the special issue of forecasting exchange rates is analyzed. A flexible neural tree is proposed for the forecasting process. Based on the pre-defined operator sets, a flexible neural tree model can be created end evolved. The structure of this technique is developed using the extended compact genetic programming and the free parameters embedded in the neural tree are optimized by particle swarm optimization algorithm.

\section{Conclusions}

As we can see from the above applications, particle swarm optimization algorithms are used in most cases. In addition, the role of these algorithms is to optimize the 
parameters of the model used to perform a certain financial task (portfolio selection, forecasting). In most cases, swarm intelligence was complementary to neural networks. However, these new nature-inspired techniques have the ability to select the optimal parameters of the model under considerations. This surely leads to better results than using traditional approaches.

Future perspectives can be divided into two categories. Firstly, there could be an improvement in technical aspects of the aforementioned methodologies. More specifically, the communication topology of the particles in the particle swarm optimization algorithm can be changed dynamically and not remain fixed. Also, the investigation of the relationship between the weighting function and the premature convergence of PSO might be a challenging task. Other, more general technical issues are the use of alternative methods or more detailed versions of the new natureinspired techniques, larger data sets (might give better estimations) etc. The second category has to do with several alternative scenarios as far as certain applications are considered. For example, in decision making problems, we can allow for more frequent trading possibilities, in the capital investment planning, more projects might be included in the company's portfolio etc.

To summarise, we can comment that nature-inspired optimization techniques might become the next step in effectively facing optimization problems. Standard methodologies sometimes lack the abilities and the efficiency of nature inspired intelligent algorithms. Moreover, nature-inspired methods can effectively be used in production, manufacturing, medical applications etc. As general future directions in the financial domain, we can indicate several problems that can be addressed by these algorithms. For example, an interesting issue is the investigation of whether there exists any relationship between national economies, stocks, rates, exchange rates etc., in other words to discover whether there exist profitable opportunities for investment.

\section{References}

1. P. C. Ko, P. C. Lin, An evolution based approach with modularized evaluations to forecast financial distress, Knowledge - Based Systems (19), 84 - 91 (2006)

2. L. Gao, C. Zhou, H.B. Gao, Y.R. Shi, Credit scoring model based on Neural Network with particle swarm optimization, Advances in Natural Computation, 76-79 (2006)

3. J. Sun, W.B. Xu, W. Fang, Solving multi-period financial planning problem via quantum - behaved particle swarm algorithm, Computational Intelligence, 1158 - 1169 (2006)

4. J. Nenortaite, R. Simutis, Development and evaluation of decision - making model for stock markets, Journal of global optimization, 1 - 19 (2006)

5. J. S. Lee, S. Lee, S. Chang, B.H. Ahn, A comparison of GA and PSO for excess return evaluation in stock markets, Artificial Intelligence and Knowledge Engineering Applications: A Bio - inspired approach, $221-230$ (2005)

6. S. S. Weng, Y. H. Liu, Mining time series data for segmentation by using Ant Colony Optimization, European Journal of Operational Research (173), 921 - 937 (2006)

7. C. Stummer, M.H. Sun, New multi - objective metaheuristic solution procedures for capital investment planning, Journal of Heuristics, $183-199$ (2005) 
8. C. Blum, Ant Colony Optimization: Introduction and recent trends, Physics of life Reviews, $353-373$ (2005)

9. E. Elmeltagi, T. Hegazy, D. Grierson, Comparison among five evolutionary - based optimization algorithms, Advanced Engineering Informatics (19), 43 - 53 (2005)

10. Y. Dong, $\mathbf{J}$. Tang, B. Xu, D. Wang, An application of swarm optimization to nonlinear programming, Computers and Mathematics with Applications (49), 1655 - 1668 (2005)

11. D.N. Jeyakumar, T. Jayabarathi, T. Raghunathan, Particle swarm optimization for various types of economic dispatch problems, Electrical Power \& Energy systems (28), 36-42 (2006)

12. M. Dorigo, L. M. Gambardella, Ant colonies for the traveling salesman problem, Biosystems 43(2),73-81 (1997)

13. M. Dorigo, V. Maniezzo, A. Colomi, Ant system: optimization by a colony of cooperating agents, IEEE Trans SystMan Cybern 26(1), 29 41(1996)

14. P. Y. Yin, Particle swarm optimization for point pattern matching, Journal of Visual Communication \& Image Representation, 143-162 (2006)

15. X. Cai, N. Zhang, G. K. Venayaagamoorthy, D. C. Wunsch II, Time series prediction with recurrent neural networks trained by a hybrid PSO-EA algorithm, Neurocomputing $70,2342-2353$ (2007)

16. Y. Chen, J. Yang, B. Yang, A. Abraham, Flexible Neural Trees Ensemble for Stock Index Modeling

17. J. Nenortaite, A particle swarm optimization approach in the construction of decisionmaking model, Information Technology and Control 1A (36) (2007)

18. G. Kendall, Y. Su, A particle swarm optimization approach in the construction of optimal risky portfolios, Artificial Intelligence and Applications (2005)

19. T. Fischer, A. Roehrl, Risk and performance optimization for portfolios of bonds and stocks (2003)

20. T. Fischer, A. Roehrl, Optimization of performance measures based on Expected Shortfall (2005)

21. J. Nenortaite, R. Simutis, Stocks' Trading System Based on the Particle Swarm Optimization Algorithm, Springer-Verlag, Heidelberg, 843-850 (2004)

22. N. G. Pavlidis, D. K. Tasoulis, M. N. Vrahatis, Financial Forecasting Through Unsupervised Clustering and Evolutionary Trained Neural Networks, IEEE (2003)

23. Y. Chen, L. Peng, A. Abraham, Exchange Rate Forecasting using Flexible Neural Networks 